as $\mathrm{E}_{6}$ was already known as a nice grand unified theory group in which the lowenergy forces fit well. Furthermore, the 15 quarks and leptons of each family fit perfectly within the 27-dimensional representation of $\mathrm{E}_{6}$. Matter and forces already known match precisely those emerging from this compactification, with a few extra forces and matter states thrown in for good measure.

The trouble is, one can have too much of a good thing. The simplest Calabi-Yau manifolds end up having enormous numbers (of the order of 100) of repetitions of the $27 \mathrm{~s}$ of quarks and leptons. They also include forces that cause protons to decay very rapidly, totally at variance with observation. A systematic investigation of more favourable manifolds has been carried out by several groups ${ }^{8-10}$ in the past year, imposing reasonable boundary conditions to weed out uninteresting manifolds from the start. One of these conditions is that the resulting group, after compactification, should be so small that none of its gauge bosons can lead directly to rapid proton decay. In effect, this requirement necessitates that the $\mathrm{E}_{6}$ group be broken at compactification. This obtains in manifolds of sufficiently complex topology so that it is possible for some of the $\mathrm{E}_{6}$ gauge fields to become trapped in holes in the manifold, leading to a symmetry breakdown ${ }^{\text {in }}$. Remarkably, precisely for these kinds of manifolds, the Euler characteristic, which details the number of matter replications, is also small. So two potential problems can be alleviated at the same time.

Two generic results emerge from these analyses $^{x-10}$. First, if all the symmetry breakdown occurs at the compactification scale, the surviving symmetry group contains the known forces plus at least one new force, whose associated gauge boson must have a mass comparable to that of the weak gauge bosons of around 100 $\mathrm{GeV}$. Furthermore, the number of families is limited to three, and exotic states in the 27 , besides quarks and leptons, survive at low energy. Second, if there are further stages of symmetry breakdown below the compactification scale, it is possible that the low-energy theory contains just the known forces. All exotic matter is, in this picture, superheavy and experimentally inaccessible; and up to four families of quarks and leptons are permitted.

Clearly the first case is more appealing phenomenologically, because it promises that both new forces and matter are there to be found, providing a 'smoking gun' to test the superstring hypothesis. However, this case is not guaranteed and is in itself not without difficulties. For instance, unless certain couplings of the exotic matter states are absent, protons will again decay rapidly. In this sense, the case with intermediate scale breaking is phenomenologically safer, but it is also almost devoid of remnant signals. This is nicely exemplified by the work of Graham Ross and his collaborators ${ }^{12}$ at Oxford, who studied in detail one of the few known three-family Calabi-Yau manifolds. At compactification, this manifold either retains the full $\mathrm{E}_{6}$ symmetry or is broken down to $\mathrm{SU}(3) \times$ $\mathrm{SU}(3) \times \mathrm{SU}(3)$ or $\mathrm{SU}(6) \times \mathrm{U}(1)$. None of these patterns is nice. However, Ross and his collaborators ${ }^{12}$ argue convincingly (although to my mind not totally compellingly) that a sequence of further breakdowns take place which reduce the SU(3) $\times \mathrm{SU}(3) \times \mathrm{SU}(3)$ theory to precisely $\mathrm{SU}(3) \times \mathrm{SU}(2) \times \mathrm{U}(1)-$ the gauge theory describing the strong and electroweak interactions. Not only are the forces standard at low energy but so is matter, with the exception of two weakly coupled neutral states. What remains as distinct predictions from the original theory are certain relationships between masses and mixing angles of quarks - predictions which are in fair agreement with experiment. Although this analysis is very nice, this is not much of a smoking gun to convince anyone who is not already an aficionado of the reality of superstrings.

In my opinion what will be necessary to sway superstring sceptics (among whom I count myself) is some hard evidence for the existence of superpartners to the lowenergy excitations we know. Supersymmetry is, after all, a key feature of superstrings and, even after the Calabi-Yau compactification it remains a good symmetry of the four-dimensional theory. Although the matter fermions divorce themselves from the gauge bosons during compactification, they pick up in the process a set of spin zero partners so that, remarkably, a supersymmetry is retained ${ }^{4}$. Eventually, below the compactification scale this supersymmetry must itself break down. How this happens is the least understood piece of the theory, although it is often suggested that the breakdown is triggered by physics associated with the other $\mathrm{E}_{8}$ group which is unaffected by the compactification. At any rate, this breakdown of supersymmetry is necessary as it is the seed which allows all 'light' masses to be generated in the theory ${ }^{\mathrm{i} 3}$, including the mass $M_{\mathrm{w}}$ of the weak gauge bosons. It is therefore natural, and in fact is required by the internal consistency of the theory, that the mass splitting among superpartners generated by this mechanism also be of the order of $M_{\mathrm{w}}$.

Superpartners with masses of the order of $100 \mathrm{GeV}$ are in the range of discovery for the forthcoming large accelerators at CERN near Geneva, DESY in Hamburg, Fermilab near Chicago and SLAC in Palo Alto. Their discovery, or that of some other debris of the superstring, would provide us with a first glimpse into extra dimensions.

1. Green, M. Nature 314, 409 (1985).

2. Georgi, H., Quinn, H. \& Weinberg, S. Phys. Rev. Lett. 33, $452(1974)$.

3. Gross, D., Harvey, J., Martinec, E \& Rohm, R. Phys. Rev. Lett. 54, 502 (1985).

4. Candelas, P., Horowitz, G., Strominger, A. \& Witten, E. Nucl. Phys. B258 46 (1985).

5. Calabi, E. in Algebraic Geometry and Topology (Princeton Univ. Press, 1957)

6. Yau, S.T. Proc. natn. Acad. Sci. U.S.A. 74, 177 (1978).

7. Dixon, L., Harvey, J.A., Vafa, C. \& Witten, E. Nucl. Phys. B261, 678 (1965)

8. Cecotti, S., Derendinger, J.P., Ferrara, S., Girardello, L. \& Roncadelli, M. Phys. Lett. 156B, 318 (1985).

9. Dine, M., Kaplunovsky, V., Nappi, C., Mangano, M. \& Seiberg, N. Nucl. Phys. B259, 549 (1985).

10. Breit, J.D., Ovrut, B. \& Segré, G. Phys. Lett. 158B, 33 (1985)

11. Witten, E. Nucl. Phys, B258, 75 (1985).

12. Greene, B. R., Kirklin, K.H., Miron, P. J. \& Ross, G. G. Phys. Lett. B180, 69 (1986)

13. Ibañez, L. Phys. Lett. 118B, 73 (1982).

Roberto Peccei is at the Deutsches Elektronen Synchrotron (DESY), 85 Notkestrasse, D-2000 Hamburg 52, FRG

\title{
Data bank for carbohydrate structures
}

THE need for an international data bank for carbohydrate structures was discussed at a recent workshop, sponsored by the US Department of Energy (Aubern, New York, 7-9 August 1986). Such a facility would provide primary structure information analogous to that obtained from the established amino-acid and nucleotide sequence data banks, which would allow the currently impossible systematic search for structural relationships between the 4,000-5,000 carbohydrate structures so far known.

The computer programs required to organize and store the carbohydrate structural data now exist but are more complex than those used for amino-acid sequences. The complexity results from the combinatorial effects of branching in the carbohydrate chain. Conference participants agreed that structural data would be collected by representatives of the different sub-fields of carbohydrate research and organized centrally at the Complex Carbohydrate Research Center, University of Georgia, under the guidance of its director Dr Peter Albersheim.

The estimated costs for the first year of the project are $\$ 200,000$. A large contribution ( 40 per cent) will be made by the US Government and the remainder will come from the twelve other participating countries. Users will pay an annual subscription of $\$ 100$. This improved access to information will be welcomed by both academic and industrial (for example, pharmaceutical, recombinant DNA, oil) concerns.

An international steering committee was set up to coordinate the growth and funding of the project and the data bank should be in full use within a year. The conclusions of the workshop will be available in about two months from the US Department of Energy.
Sarah Hargreaves 